\title{
FRATURA DISTAL DO RÁDIO EM PACIENTES COM MAIS DE 60 ANOS: PLACAS ORTOGONAIS VERSUS PLACA VOLAR
}

\author{
DISTAL RADIAL FRACTURES IN PATIENTS OVER \\ 60 YEARS OLD: ORTHOGONAL PLATES VERSUS VOLAR PLATE
}

Edgard Novaes de França Bisneto', Emygdio José Leomil de Paula', Marcelo Rosa de Resende', Rames Mattar Júnior ${ }^{2}$, Arnaldo Valdir Zumiotti ${ }^{3}$

\section{RESUMO}

Objetivo: Comparar os resultados do tratamento cirúrgico entre placa volar com estabilidade angular e placas ortogonais em fraturas instáveis de rádio distal em pacientes com mais de 60 anos. Métodos: Pacientes foram divididos em dois grupos tratados com placa volar ou placas ortogonais. Resultados clínicos e radiográficos foram analisados prospectivamente. Resultados: Os grupos de estudo apresentaram resultados clínicos e radiográficos semelhantes seis meses após a operação. No entanto três meses após a cirurgia, o grupo onde foi utilizada a placa volar obteve resultados superiores. Conclusão: Ambos os grupos apresentaram bons resultados funcionais. O tratamento cirúrgico facilita a reabilitação precoce. A técnica das placas ortogonais requer uma curva de aprendizado maior e apresentou mais complicações e piores resultados iniciais.

Descritores - Fraturas do Rádio Distal; Placas Volares; Placas Ortogonais; Fraturas em Idosos

\section{ABSTRACT:}

Objective: To compare the results from surgical treatment between volar plates with angular stability and orthogonal plates of unstable distal radius fractures in patients aged over 60 years. Methods: Selected patients were randomized in two groups treated with volar plates or orthogonal plates. Clinical and radiographic results were analyzed prospectively. Results: The study groups presented similar clinical and radiographic results six months after the operation. However three months after surgery the volar plate group obtained superior results considering most of the parameters studied. Conclusion: Both the volar plates and the orthogonal plates presented good functional results. Surgical treatment enabled early rehabilitation for these patients. Orthogonal plate technique required a longer learning curve, presented more complications and worst early results.

Keywords - Wrist Injuries; Distal Radius Fractures; Fractures in Elderly; Volar Plates; Orthogonal Plates

\section{INTRODUÇÃO}

Apesar das mudanças na expectativa e qualidade de vida de pacientes acima de 60 anos de idade, as fraturas da extremidade distal do radio nesta população ainda são, frequentemente, negligenciadas ${ }^{(1)}$.

Claramente a população mundial vem envelhecendo, com as expectativas médias de vida aumentando, inclusive no Brasil, principalmente nos grandes centros urbanos (fonte: IBGE). Paralelamente a este fato, observa-se que a qualidade de vida do idoso também melhorou nestes últimos anos, com várias pessoas com mais de 60 anos praticando exercícios físicos regularmente. Portanto, entre os critérios adotados para indicações terapêuticas destas fraturas, a idade cronológica vem perdendo importância frente à análise do estado geral do paciente e de suas expectativas quanto a recuperação funcional ${ }^{(1)}$.

A utilização de fixadores externos, apesar de bons resultados, tem sido questionada em relação ao uso de placas com estabilidade angular, para a fixação

\footnotetext{
1 - Médico Assistente Doutor do Instituto de Ortopedia e Traumatologia do HC-FMUSP.

2 - Professor Associado da Faculdade de Medicina da Universidade de São Paulo.

3 - Professor Titular do Departamento de Ortopedia e Traumatologia da FMUSP.
}

Trabalho realizado no LIM 41 - Laboratório de Investigação Médica do Sistema Musculoesquelético do Departamento de Ortopedia e Traumatologia.

Correspondência: Rua Tucuna, 10 - apto. 41 - VI. Pompéia. São Paulo - SP - 05021-010. E-mail: novaesf@terra.com.br

Trabalho recebido para publicação: 17/03/10, aceito para publicação: 12/07/10. 
específica de fragmentos, principalmente em fraturas intra-articulares, pois, com a placa o retorno funcional seria mais precoce ${ }^{(2,3)}$.

A grande vantagem de proporcionar retorno mais precoce às atividades de vida diária tem sido um argumento que defende a utilização de placas volares com estabilidade angular. Estas placas são capazes de manter a redução mesmo em ossos osteoporóticos ${ }^{(4-6)}$.

O uso de placas dorsais tem sido relacionado com complicações tendíneas. As complicações com os tendões extensores são frequentes a ponto de autores recomendarem sua retirada de rotina após a consolidação da fratura ${ }^{(7)}$.

A análise biomecânica dos dois métodos de fixação revelam equivalência de resistência, havendo uma pequena vantagem da fixação específica de fragmentos com placas ortogonais ${ }^{(8)}$.

Este estudo prospectivo objetiva analisar os resultados funcionais e radiográficos entre pacientes portadores de fraturas do terço distal do rádio, maiores de 60 anos, submetidos a duas técnicas cirúrgicas distintas.

\section{MATERIAL E MÉTODO}

Foram incluídos neste estudo 19 pacientes com de idades variando de 60 a 87 anos, sendo 17 pacientes do sexo feminino e dois do sexo masculino, portadores de fraturas da extremidade distal do rádio intra-articulares tipos B/C pela classificação do grupo AO-ASIF. Os pacientes foram divididos de forma randomizada por meio de sorteio com moeda em dois grupos:

- Grupo A: tratados com placa volar com abordagem volar pela via descrita por Henry e colocação de placa LCP 3,5mm (Synthes Ind. e Com. Ltda.) (Figuras 1 e 2).

- Grupo B: tratados com duas placas de 2,4mm (Synthes Ind. e Com. Ltda.) dispostas ortogonalmente através de duas vias cirúrgicas, uma lateral e outra dorsal, fixando fragmentos específicos (Figuras 3 e 4).

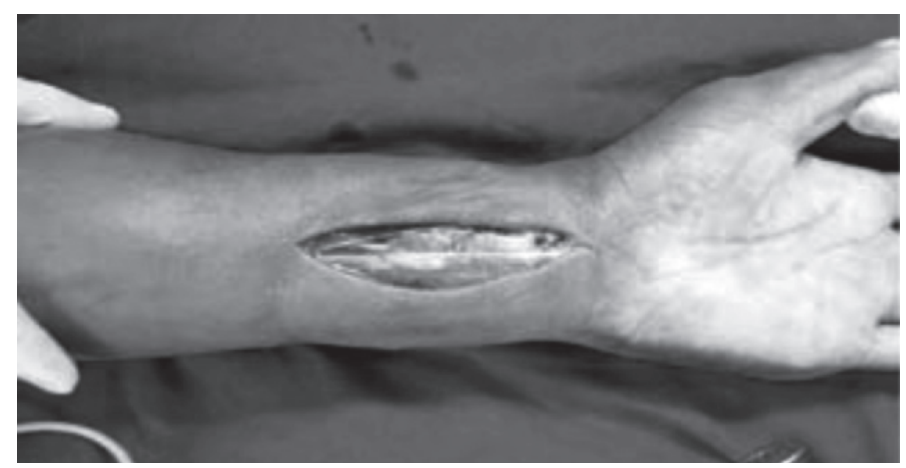

Figura 1 - Incisão volar tipo Henry para a colocação da placa volar.

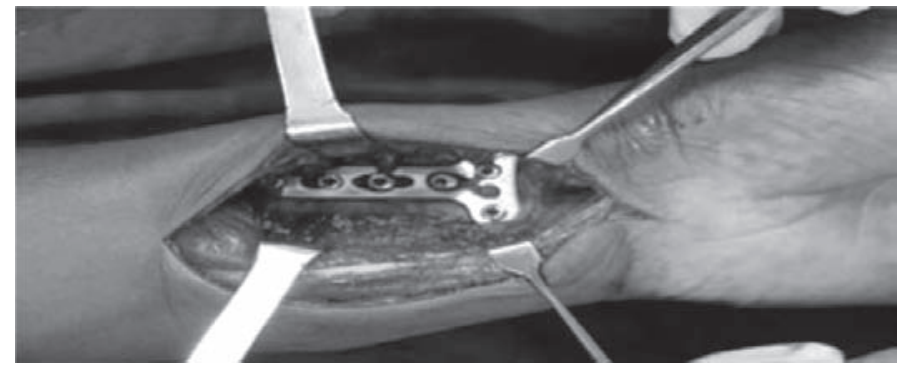

Figura 2 - Placa volar (Synthes 3,5mm) colocada.

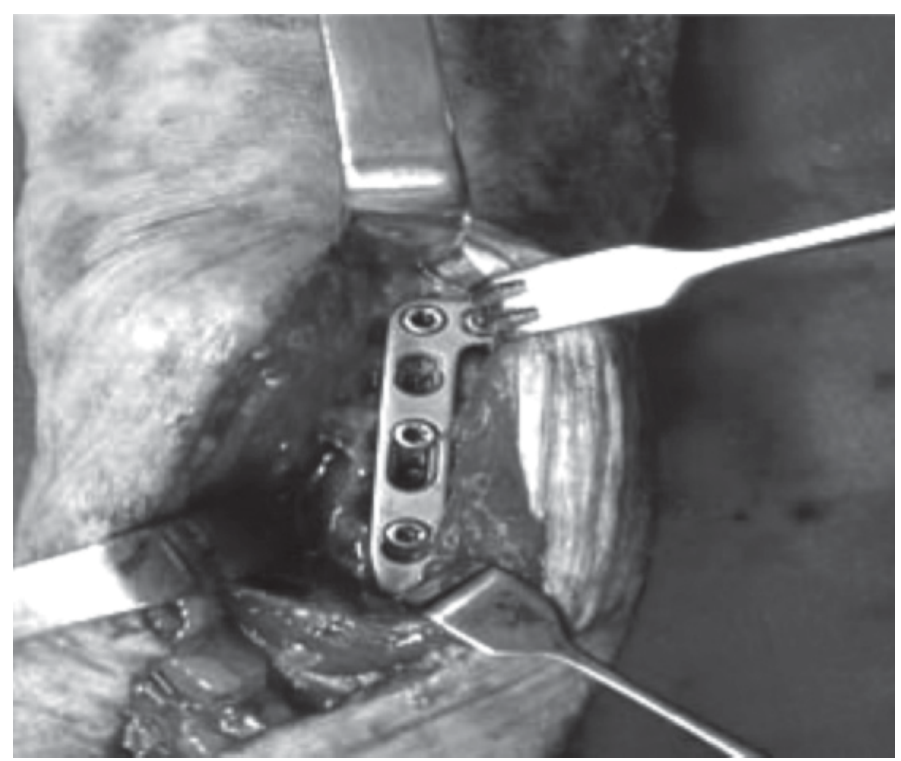

Figura 3 - Placa (Synthes 2,7mm) colocado entre o quarto e 0 quinto compartimentos extensores.

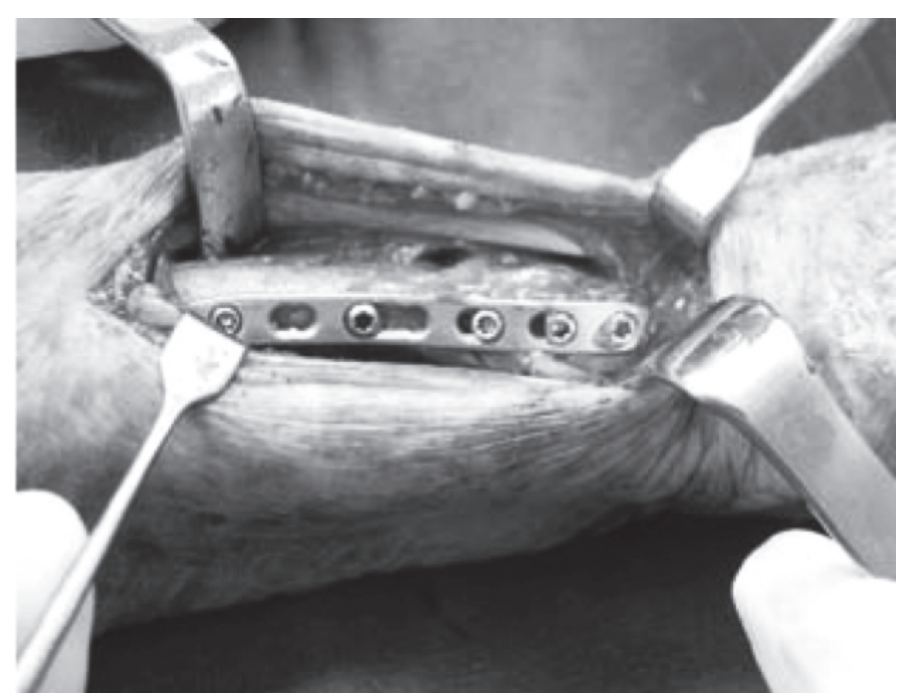

Figura 4 - Placa (Synthes 2,7mm) colocada sob o primeiro compartimento.

Cada paciente foi avaliado no pós-operatório imediato e com três e seis meses após a cirurgia. A avaliação consistiu na análise radiográfica, goniometria e teste de força de preensão $\left(\operatorname{Jamar}^{\circledR}\right)$. 
A reabilitação iniciada entre sete a 10 dias de pósoperatório, quando a tala gessada era trocada por uma órtese de material termoplástico. A movimentação ativa dos dedos era incentivada no pós-operatório imediato. A partir de quatro semanas os pacientes iniciavam exercícios contrarresistência e as órteses eram retiradas após seis semanas (Figura 5).

As análises estatísticas foram realizadas por modelos de medidas repetidas com fator aleatório interindivíduo, referente às quatro mensurações em cada paciente, e um fator fixo entre indivíduos, referente ao tratamento placas volares ou ortogonais. As dependências entre as mensurações realizadas nos mesmos pacientes foram incorporadas com matrizes de covariâncias não estruturadas, com exceção da medida da pronação em que foi utilizada a estrutura compound symmetry. Foram consideradas diferenças significativas os níveis descritivos (p) menores ou iguais a 5\%.

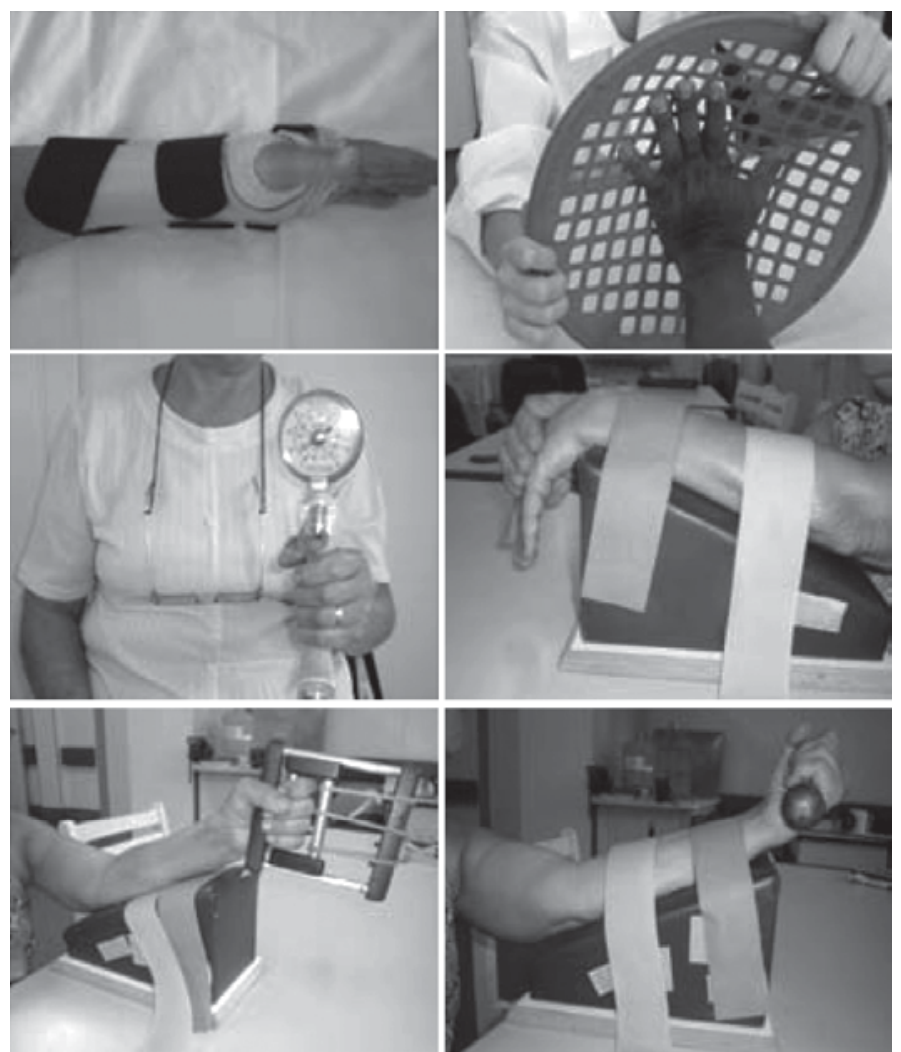

Figura 5 - Exercícios de reabilitação iniciados no $10^{\circ}$ dia de pós-operatório.

\section{RESULTADOS}

A média de idade nos grupos foi semelhante: 73,16 anos no grupo A, submetido às placas volares e 74,25 anos no grupo $\mathrm{B}$, submetido às placas ortogonais.

Como um dos parâmetros de avaliação foi a comparação com o punho não operado, comparamos a domi- nância entre os grupos, o que também foi semelhante. No grupo A 58\% $(n=7)$ dos pacientes que sofreram a fratura do rádio no membro dominante; já no grupo B, também houve prevalência no membro dominante, $62 \%$ $(\mathrm{n}=5)$ das pacientes.

Na Tabela 1 observamos os dados obtidos na goniometria e avaliação de força dos pacientes do grupo A.

Tabela 1 - Avaliação funcional grupo A.

\begin{tabular}{c|c|c|c|c}
\hline & \multicolumn{4}{|c}{ Placas Volares } \\
\cline { 2 - 5 } & $\begin{array}{c}\text { Lado } \\
\text { contralateral } \\
(11)\end{array}$ & 1 mês (12) & 3 meses (11) & 6 meses (08) \\
\hline Flexão & 64,18 & 37,66 & 49,63 & 54,12 \\
\hline Extensão & 57,7 & 38,33 & 51,09 & 50,62 \\
\hline DU & 30,36 & 20,3 & 27,9 & 27,62 \\
\hline DR & 19,09 & 12,75 & 20,09 & 20 \\
\hline PRON & 77,27 & 73,33 & 76,81 & 79,37 \\
\hline SUP & 87,27 & 74,58 & 84,54 & 83,12 \\
\hline $\begin{array}{c}\text { Força Kg/F } \\
\text { (Jamar) }\end{array}$ & 21,02 & 6,11 & 10,7 & 13,7 \\
\hline
\end{tabular}

Na Tabela 2 observamos os dados obtidos na goniometria e avaliação de força dos pacientes do grupo B.

Tabela 2 - Avaliação funcional grupo B.

\begin{tabular}{c|c|c|c|c}
\hline & \multicolumn{4}{|c}{ Placas Ortogonais } \\
\hline /graus & $\begin{array}{c}\text { Lado } \\
\text { contralateral } \\
(08)\end{array}$ & 1 mês (08) & 3 meses (7) & 6 meses (5) \\
\hline Flexão & 67,5 & 25,62 & 36,85 & 57 \\
\hline Extensão & 58,62 & 32,12 & 48,71 & 55 \\
\hline DU & 28,62 & 14,75 & 21,57 & 26,6 \\
\hline DR & 17 & 7,5 & 12,5 & 16,4 \\
\hline PRON & 80,62 & 75 & 78,57 & 80 \\
\hline SUP & 86,87 & 67,5 & 80,71 & 81 \\
\hline $\begin{array}{c}\text { Força Kg/F } \\
\text { (Jamar) }\end{array}$ & 16,17 & 4,33 & 7,5 & 12 \\
\hline
\end{tabular}

Nas Tabelas 3 e 4, a evolução relativa das médias dos dados obtidos por goniometria e das médias de força de preensão em comparação as médias dos lados não operados

Houve três graves complicações. Duas rupturas tendíneas, ELP em uma paciente e ECD em outra paciente e um caso de distrofia simpaticorreflexa. Todos estes pacientes pertencem ao grupo $\mathrm{B}$, de placas ortogonais. Nos dois primeiros pacientes foram retiradas as placas dorsais e realizado o tratamento adequado para a lesão 
Tabela 3 - Avaliação funcional comparativa entre o lado operado e o contralateral no grupo B.

\begin{tabular}{c|c|c|c}
\hline \multirow{2}{*}{} & \multicolumn{3}{|c}{ Placas Ortogonais } \\
\cline { 2 - 4 } & Percentual evolutivo comparado ao lado não operado \\
\hline /graus & 1 mês & 3 meses & 6 meses \\
\hline Flexão & $38 \%$ & $55 \%$ & $84 \%^{*}$ \\
\hline Extensão & $55 \%$ & $83 \%$ & $94 \%^{*}$ \\
\hline DU & $52 \%$ & $75 \%$ & $93 \%$ \\
\hline DR & $44 \%$ & $74 \%$ & $96 \%$ \\
\hline PRON & $93 \%$ & $97 \%$ & $99 \%$ \\
\hline SUP & $78 \%$ & $93 \%$ & $93 \%{ }^{*}$ \\
\hline $\begin{array}{c}\text { Força Kg/F } \\
\text { (Jamar) }\end{array}$ & $27 \%$ & $46 \%$ & $74 \%{ }^{*}$ \\
\hline
\end{tabular}

Tabela 4 - Avaliação funcional comparativa entre o lado operado e o contralateral no grupo A.

\begin{tabular}{c|c|c|c}
\hline \multirow{2}{*}{} & \multicolumn{3}{|c}{ Placas Volares } \\
\cline { 2 - 4 } & \multicolumn{3}{|c}{ Percentual evolutivo comparado ao lado não operado } \\
\hline /graus & 1 mês & 3 meses & 6 meses \\
\hline Flexão & $59 \%$ & $77 \%$ & $84 \%^{*}$ \\
\hline Extensão & $66 \%$ & $89 \%$ & $88 \%^{*}$ \\
\hline DU & $67 \%$ & $92 \%$ & $91 \%$ \\
\hline DR & $67 \%$ & $105 \%$ & $105 \%$ \\
\hline PRON & $95 \%$ & $99 \%$ & $103 \%$ \\
\hline SUP & $85 \%$ & $97 \%$ & $95 \%$ \\
\hline $\begin{array}{c}\text { Força Kg/F } \\
\text { (Jamar) }\end{array}$ & $29 \%$ & $51 \%$ & $65 \%{ }^{*}$ \\
\hline
\end{tabular}

tendínea. No terceiro caso, a paciente recuperou-se com tratamento clínico. Ainda houve um quarto paciente, também com placas ortogonais, que foi submetido à retirada das placas por presença de um parafuso intraarticular. Não houve casos de infecção.

Durante o estudo observamos que em alguns pacientes houve o que chamamos de “acomodação” da fratura, principalmente nos casos, nos quais, o desvio inicial da fratura era dorsal. Esta "acomodação" foi notada pela consequente perda dos parâmetros de redução iniciais: inclinação volar, inclinação ulnar e variância ulnar. Estes dados não foram submetidos a análise estatística devido as dificuldades de padronização das radiografias e pelo baixo número de pacientes, sendo considerados como dados observacionais.

Nas Tabelas 5 e 6 os valores médios obtidos nas avaliações radiográficas de 12 casos deste estudo, divididos entre os grupos A e B. Os valores negativos correspondem à inversão dos parâmetros tidos como normais.
Tabela 5 - Avaliação dos casos onde houve alteração de parâmetros radiográficos no grupo $A$.

\begin{tabular}{c|c|c|c}
\hline \multirow{2}{*}{} & \multicolumn{3}{|c}{ Placas Volares } \\
\cline { 2 - 4 } & \multicolumn{3}{|c}{ média dos valores encontrados em sete casos } \\
\cline { 2 - 4 } & POI & 3 meses & Média de perda \\
\hline Angulação radial & $22^{\circ}$ & $15^{\circ}$ & $7^{\circ}$ \\
\hline Variância ulnar & $2 \mathrm{~mm}$ & $1 \mathrm{~mm}$ & $1 \mathrm{~mm}$ \\
\hline Inclinação volar & $3^{\circ}$ & $0^{\circ}$ & $3^{\circ}$ \\
\hline
\end{tabular}

Tabela 6 - Avaliação dos casos onde houve alteração de parâmetros radiográficos no grupo $B$.

\begin{tabular}{c|c|c|c}
\hline \multirow{2}{*}{} & \multicolumn{3}{|c}{ Placas Ortogonals } \\
\cline { 2 - 4 } & \multicolumn{2}{|c|}{ média dos valores encontrados em cinco casos } \\
\cline { 2 - 4 } & $\mathrm{POI}$ & 3 meses & Média de perda \\
\hline Angulação radial & $21^{\circ}$ & $12^{\circ}$ & $9^{\circ}$ \\
\hline Variância ulnar & $1 \mathrm{~mm}$ & $+1 \mathrm{~mm}$ & $2 \mathrm{~mm}$ \\
\hline Inclinação volar & $-3^{\circ}$ & $-7.5^{\circ}$ & $4.5^{\circ}$ \\
\hline
\end{tabular}

Da mesma forma pudemos constatar que os valores, em média, obtidos ao final do estudo, são estatisticamente significantes. A exceção está nos valores obtidos para pronação que não se mostraram estatisticamente significante em nenhuma das avaliações (Tabela 7).

Tabela 7 - Comparação funcional entre os grupos durante a evolução.

\begin{tabular}{c|c|c|c|c|c|c}
\hline \multirow{2}{*}{ /graus } & \multicolumn{3}{|c}{ Análise comparativa da evolução entre os grupos } \\
\hline & $\begin{array}{c}1 \text { mês de } \\
\text { avaliação }\end{array}$ & \multicolumn{2}{c}{$\begin{array}{c}3 \text { meses de } \\
\text { avaliação }\end{array}$} & \multicolumn{2}{c}{$\begin{array}{c}6 \text { meses de } \\
\text { avaliação }\end{array}$} \\
\hline \multirow{2}{*}{ Plexão } & $59 \%$ & $38 \%$ & $77 \%$ & $55 \%$ & $84 \%$ & $84 \%^{*}$ \\
\hline Extensão & $66 \%$ & $55 \%$ & $89 \%$ & $83 \%$ & $88 \%$ & $94 \%^{*}$ \\
\hline DU & $67 \%$ & $52 \%$ & $92 \%$ & $75 \%$ & $91 \%$ & $93 \%{ }^{*}$ \\
\hline DR & $67 \%$ & $44 \%$ & $105 \%$ & $74 \%$ & $105 \%$ & $96 \%{ }^{*}$ \\
\hline PRON & $95 \%$ & $93 \%$ & $99 \%$ & $97 \%$ & $103 \%$ & $99 \%$ \\
\hline SUP & $85 \%$ & $78 \%$ & $97 \%$ & $93 \%$ & $95 \%$ & $93 \%$ \\
\hline $\begin{array}{c}\text { Força Kg/F } \\
\text { (Jamar) }\end{array}$ & $29 \%$ & $27 \%$ & $51 \%$ & $46 \%$ & $65 \%$ & $74 \% *$ \\
\hline
\end{tabular}

Em relação às perdas observadas na evolução radiográfica, os valores, em média obtidos, não só são semelhantes como também não influenciaram nos resultados funcionais (Tabela 8).

\section{DISCUSSÃO}

Leibovic e Geissler ${ }^{(9)}$ demonstram que o tratamento cirúrgico com fixação adequada do terço distal do rá- 
Tabela 8 - Avaliação comparativa das alterações dos parâmetros radiográficos.

\begin{tabular}{c|c|c}
\hline & Média de Perda & Média de Perda \\
\hline & Placa volar & Placa ortogonal \\
\hline Angulação radial & $7^{\circ}$ & $9^{\circ}$ \\
\hline Variância ulnar & $1 \mathrm{~mm}$ & $2 \mathrm{~mm}$ \\
\hline Inclinação volar & $3^{\circ}$ & $4.5^{\circ}$ \\
\hline
\end{tabular}

dio, associado à reabilitação precoce, principalmente nas fraturas intra-articulares, apresenta melhor resultado quando comparado ao tratamento conservador.

Ring $^{(1)}$ observa que os idosos evoluem muitas vezes para dor e restrição do arco de movimento (ADM), com perda da qualidade de vida, além de complicações que eventualmente podem acontecer devido ao mau posicionamento do rádio, entre eles: artrose radiocárpica e artrose da articulação rádio-ulnar distal.

Harness et $a^{(10)}$ observam que, mesmo fraturas relativamente simples em adultos jovens, quando incidem em pacientes mais idosos mudam suas características, apresentando-se com maior cominuição, o que as torna potencialmente mais graves e com maior chance de evoluírem para artrose. Esta observação corrobora os achados de Hegeman et al ${ }^{(11)}$ segundo os quais $51 \%$ dos pacientes portadores da fratura da extremidade distal do rádio possuíam osteoporose e $81 \%$ osteopenia. Hollevoet et $a l^{(12)}$ classificam a diminuição da densidade mineral óssea como fator de gravidade nas fraturas do terço distal do rádio.

As fraturas do terço distal do rádio em idosos, na grande maioria dos casos, eram tratadas conservadoramente a despeito de sua gravidade pelo fato de considerar o idoso um paciente com baixa demanda funcional analisando-se somente a idade cronológica ${ }^{(1)}$. Claramente, ao generalizarmos desta forma, incorreremos em um erro. Todos os pacientes possuem suas próprias características. Entretanto, uma destas características tende a ser comum a todos: a diminuição da densidade mineral óssea ${ }^{(11,12)}$. Este fato tornou o tratamento cirúrgico dos pacientes idosos um desafio ao cirurgião, pois a qualidade óssea não permitia uma fixação ideal dos fragmentos ósseos ${ }^{(13)}$.

O tratamento conservador nas fraturas instáveis geralmente evoluem com perda da redução, McQueen et $a l^{(14)}$ analisaram 120 fraturas da extremidade distal do rádio inicialmente tratadas conservadoramente que evoluíram para perda da redução. Neste estudo, os autores comparam a recolocação de gesso pós-redução incruen- ta, enxerto ósseo com fixação por meio de fios de Kirschner e fixador externo mantido três e seis semanas. Em nenhum dos métodos houve melhora do alinhamento carpal, principalmente nas fraturas instáveis.

Hegeman et $a^{(15)}$ também utilizaram o fixador externo como forma de tratamento em pacientes acima de 55 anos portadores de fratura da extremidade distal do rádio. Neste estudo, dos 16 pacientes operados, 11 perderam a redução com o fixador externo e cinco evoluíram com distrofia simpaticorreflexa.

As placas e parafusos convencionais não se mostravam adequadas ao osso desmineralizado, pois sua ação fundamentada no atrito entre placa e osso e fixação dos parafusos baseada nas corticais ósseas é ruim no osso osteopênico.

Vários estudos sobre a utilização de placas dorsais foram publicados ${ }^{(13,16-19)}$. A redução mantida com as placas dorsais é descrita em vários trabalhos; entretanto, em todas as grandes séries nas quais se utilizou este método, encontramos complicações referentes aos tendões extensores. Herron et $a^{(18)}$ descreveram que, de 30 pacientes operados, 11 retiraram a placa devido a complicações com os tendões extensores e um paciente sofreu efetivamente uma ruptura total ${ }^{(16)}$. Beharrie et al $^{(16)}$ descreveram um caso de tendinite extensora justamente em associação à placa dorsal.

A utilização das placas volares de ângulo fixo, isto é, com estabilidade angular para o tratamento das fraturas do rádio distal foi popularizada principalmente por Orbay e Fernandez ${ }^{(6,20,21)}$. A técnica de placas ortogonais, com placas de 2,4mm, também com estabilidade angular, foi descrita e popularizada por Peine et $a^{(17)}$.

Neste estudo observamos um predomínio feminino, pois 89,5\% (17 de 19) dos pacientes são mulheres. Este fato corrobora os dados da literatura que atribuem as fraturas da extremidade distal do rádio um sinal importante de osteoporose, que ocorre de forma mais rápida e intensa nas mulheres ${ }^{(11,12)}$.

Nenhum dos pacientes demonstrou qualquer problema de rigidez de cotovelo ou dor nos ombros, comuns no tratamento conservador destas fraturas em idosos. Não houve casos de infecção. Não houve intercorrências clínicas importantes.

Comparando os grupos, temos 12 punhos no grupo A contra oito no grupo B. Esta variação se deu pelo fato do método de randomização escolhido. Entretanto, podemos observar semelhança na média de idade e na dominância do membro afetado: 73,1 anos contra 74,2 anos e 58\% contra $62 \%$ dos membros dominantes afetados. 
Analisando a evolução goniométrica e de força de preensão dos pacientes nos dois grupos podemos observar claramente uma melhor avaliação, estatisticamente significante a favor das placas volares no primeiro e no terceiro mês de evolução.

A utilização de placas orgonais para fixação específica de fragmentos causou complicações consideradas de grande morbidade como a ruptura de tendões extensores em dois pacientes, presença de parafuso intra-articular em um paciente e distrofia simpaticorreflexa em um paciente. Por outro lado, não observamos complicações desta natureza com a utilização das placas volares.

A grande maioria dos pacientes mostraram-se satisfeitos com o tratamento, lembrando que esta avaliação foi pregressa às complicações mais graves supracitadas. Observamos que, independente do grupo estudado, os pacientes com um mês de pós-operatório já apresentavam boa função.

\section{REFERÊNCIAS}

1. Ring D. Treatment of the neglected distal radius fracture. Clin Orthop Relat Res. 2005;(431):85-92.

2. Ochman S, Frerichmann U, Armsen N, Raschke MJ, Meffert RH. [Is use of the fixateur externe no longer indicated for the treatment of unstable radial fracture in the elderly?]. Unfallchirurg. 2006;109(12):1050-7.

3. Benson LS, Minihane KP, Stern LD, Eller E, Seshadri R. The outcome of intraarticular distal radius fractures treated with fragment-specific fixation. J Hand Surg Am. 2006;31(8):1333-9.

4. Voigt $\mathrm{C}$, Lill H. [What advantages does volar plate fixation have over K-wire fixation for distal radius extension fractures in the elderly?]. Unfallchirurg. 2006;109(10):845-6.

5. Gruber G, Bernhardt GA, Köhler G, Gruber K. Surgical treatment of distal radius fractures with an angle fixed bar palmar plating system: a single center study of 102 patients over a 2-year period. Arch Orthop Trauma Surg. 2006;126(10):680-5.

6. Orbay J, Badia A, Khoury RK, Gonzalez E, Indriago I. Volar fixed-angle fixation of distal radius fractures: the DVR plate. Tech Hand Up Extrem Surg. 2004;8(3):142-8.

7. Keller M, Steiger R. [Open reduction and internal fixation of distal radius extension fractures in women over 60 years of age with the dorsal radius plate (pi-plate)]. Handchir Mikrochir Plast Chir. 2006;38(2):82-9.

8. Taylor KF, Parks BG, Segalman KA. Biomechanical stability of a fixed-angle volar plate versus fragment-specific fixation system: cyclic testing in a C2-type distal radius cadaver fracture model. J Hand Surg Am. 2006;31(3):373-81.

9. Leibovic SJ, Geissler WB. Treatment of complex intra-articular distal radius fractures. Orthop Clin North Am. 1994;25(4):685-706.

10. Harness N, Ring D, Jupiter JB. Volar Barton's fractures with concomitant dorsal fracture in older patients. J Hand Surg Am. 2004;29(3):439-45.

11. Hegeman JH, Oskam J, van der Palen J, Ten Duis HJ, Vierhout PA. The distal radial fracture in elderly women and the bone mineral density of the lumbar
Os autores concordam que a técnica cirúrgica das placas volares é mais simples, com menor agressão de partes moles e com menor tempo de cirurgia.

\section{CONCLUSÃO}

Acreditamos que o tratamento cirúrgico no paciente idoso é a melhor opção no que diz respeito às fraturas instáveis do terço distal do rádio. Entre os grupos estudados, os autores consideram as placas volares mais seguras por possuírem menos complicações. Funcionalmente as técnicas mostraram-se semelhantes após seis meses, entretanto o primeiro e no terceiro mês de pós-operatório houve valores estatisticamente significantes favoráveis ao grupo das placas volares. A técnica das placas ortogonais exige uma curva de aprendizado maior e deve ser utilizada ao nosso modo de ver em casos selecionados. spine and hip. J Hand Surg Br. 2004;29(5):473-6.

12. Hollevoet $N$, Verdonk $R$. Outcome of distal radius fractures in relation to bone mineral density. Acta Orthop Belg. 2003;69(6):510-4.

13. Duncan SF, Weiland AJ. Minimally invasive reduction and osteosynthesis of articular fractures of the distal radius. Injury. 2001;32(Suppl 1):SA14-24.

14. McQueen MM, Hajducka C, Court-Brown CM. Redisplaced unstable fractures of the distal radius: a prospective randomised comparison of four methods of treatment. J Bone Joint Surg Br. 1996;78(3):404-9.

15. Hegeman JH, Oskam J, Vierhout PA, Ten Duis HJ. External fixation for unstable intra-articular distal radial fractures in women older than 55 years. Acceptable functional end results in the majority of the patients despite significant secondary displacement. Injury. 2005;36(2):339-44.

16. Beharrie AW, Beredjiklian PK, Bozentka DJ. Functional outcomes after open reduction and internal fixation for treatment of displaced distal radius fractures in patients over 60 years of age. J Orthop Trauma. 2004;18(10):680-6.

17. Peine R, Rikli DA, Hoffmann R, Duda G, Regazzoni P. Comparison of three different plating techniques for the dorsum of the distal radius: a biomechanical study. J Hand Surg Am. 2000;25(1):29-33.

18. Herron M, Faraj A, Craigen MA. Dorsal plating for displaced intra-articular fractures of the distal radius. Injury. 2003;34(7):497-502.

19. Ring D. Intra-articular fractures of the distal-radius. J Am Soc Surg Hand. 2002;2:60-77.

20. Orbay JL, Fernandez DL. Volar fixation for dorsally displaced fractures of the distal radius: a preliminary report. J Hand Surg Am. 2002;27(2):205-15.

21. Orbay JL, Fernandez DL. Volar fixed-angle plate fixation for unstable distal radius fractures in the elderly patient. J Hand Surg Am. 2004;29(1):96-102.

22. Osada D, Viegas SF, Shah MA, Morris RP, Patterson RM. Comparison of differentdistal radius dorsal and volar fracture fixation plates: a biomechanical study. J Hand Surg Am. 2003;28(1):94-104. 